University of Nebraska - Lincoln

DigitalCommons@University of Nebraska - Lincoln

\title{
Effects of Cognitive Behavioral Treatment on Physical Health Status in Patients With Panic Disorder
}

\author{
Norman B. Schmidt \\ Ohio State University \\ Beth T. McCreary \\ Johns Hopkins Bloomberg School of Public Health \\ John J. Trakowski \\ Uniformed Services University of the Health Sciences \\ Helen T. Santiago \\ Uniformed Services University of the Health Sciences \\ Kelly Woolaway-Bickel \\ Ohio State University \\ See next page for additional authors
}

Follow this and additional works at: https://digitalcommons.unl.edu/usuhs

Part of the Medicine and Health Sciences Commons

Schmidt, Norman B.; McCreary, Beth T.; Trakowski, John J.; Santiago, Helen T.; Woolaway-Bickel, Kelly; and lalongo, Nicholas, "Effects of Cognitive Behavioral Treatment on Physical Health Status in Patients With Panic Disorder" (2003). Uniformed Services University of the Health Sciences. 26.

https://digitalcommons.unl.edu/usuhs/26

This Article is brought to you for free and open access by the U.S. Department of Defense at DigitalCommons@University of Nebraska - Lincoln. It has been accepted for inclusion in Uniformed Services University of the Health Sciences by an authorized administrator of DigitalCommons@University of Nebraska Lincoln. 
Authors

Norman B. Schmidt, Beth T. McCreary, John J. Trakowski, Helen T. Santiago, Kelly Woolaway-Bickel, and Nicholas lalongo 


\title{
Effects of Cognitive Behavioral Treatment on Physical Health Status in Patients With Panic Disorder
}

\author{
NORMAN B. SCHMIDT \\ The Ohio State University \\ BETH T. MCCREARY
}

Johns Hopkins Bloomberg School of Public Health

JOHN J. TRAKOWSKI

Helen T. Santiago

Uniformed Services University of the Health Sciences

Kelly WoOlaWay-Bickel

The Ohio State University

NiCHOLAS IALONGO

Johns Hopkins Bloomberg School of Public Health

\begin{abstract}
The relationship between panic disorder and nonpsychiatric medical illness is complex, but some evidence suggests that panic disorder increases risk for a variety of nonpsychiatric medical conditions. Given the demonstrated efficacy of cognitive behavioral therapy (CBT) for panic disorder, we were interested in evaluating the effects of CBT for panic disorder on nonpsychiatric medical symptoms among these patients. Patients were randomized to a 12-week group-administered CBT protocol $(n=22)$ or a delayedtreatment control $(n=24)$. Treated patients showed marked improvement in both anxiety symptoms and physical health symptom ratings that were evident at midtreatment and were maintained through a 6-month follow-up period. Despite comparable rates of change, changes in anxiety symptoms did not appear to mediate the relationship between treatment and improved physical health ratings. These findings indicate that CBT appears to have an immediate and long-term beneficial impact on physical health and that this effect is independent from its impact on anxiety symptoms.
\end{abstract}

This research was supported by USUHS Grant RO72CF from the Uniformed Services University of the Health Sciences. The authors thank. Jan Kiecolt-Glaser for her comments on an earlier version of this manuscript.

Address correspondence to Norman B. Schmidt, The Ohio State University, Department of Psychology, 216 Townshend Hall, 1885 Neil Avenue Mall, Columbus, OH 43210-1222; e-mail: schmidt.283@osu.edu. 
Epidemiological reports indicate that a variety of medical conditions are more prevalent in those with a lifetime history of an anxiety disorder (Wells, Golding, \& Burnam, 1989). Panic disorder in particular is frequently complicated by the presence of nonpsychiatric medical conditions. The association between panic disorder and cardiorespiratory disorders such as asthma, chronic obstructive pulmonary disease, and mitral valve prolapse is well established (Gorman et al., 1988; Karajgi, Rifkin, Doddi, \& Kolli, 1990; Weissman, Markowitz, Ouellette, Greenwald, \& Kahn, 1990; Zandbergen et al., 1991).

The relationship between panic disorder and nonpsychiatric medical illness is complex. It appears that nonpsychiatric medical conditions can contribute to the development of panic disorder (Kahn, Drusin, \& Klein, 1987; Raj, Corvea, \& Dagon, 1993) and/or exacerbate panic disorder symptoms (McCue \& McCue, 1984). In addition, increasing evidence suggests that panic disorder can contribute to the development of physical conditions and/or exacerbate existing physical conditions (Karajgi et al., 1990; Kawachi et al., 1994). For example, longitudinal evaluation of panic disorder indicates an increased risk for a number of medical conditions (e.g., hypertension, migraine headaches, ulcer, thyroid disease) compared to patients with other anxiety conditions and the general population (Rogers et al., 1994).

The effect of stress on the immune system is one possible pathway to explain the relationship between anxiety and physical health. Stress is frequently associated with changes in immune parameters (Cacioppo, 1993; Glaser et al., 1990). Moreover, stress has been linked to increases in infectious illness (Kiecolt-Glaser \& Glaser, 1995). Thus, chronic and debilitating conditions such as panic disorder are likely to compromise immune functioning. Findings from immune studies of patients with panic disorder are somewhat mixed, though alterations in immune measures have been noted (Andreoli et al., 1992; Coplan et al., 1999; Marazziti et al., 1992; Rapaport, 1998; Rapaport \& Stein, 1994). However, we are not aware of studies that have evaluated whether panic disorder also increases risk for infectious illness.

Fortunately, there are effective means of treating panic disorder. Pharmacological and psychosocial treatments have been found to be efficacious (Wolfe \& Maser, 1994). In particular, cognitive behavioral therapy (CBT) for panic disorder has been found to be extremely effective in ameliorating panicrelated symptoms using both individual (Barlow, Craske, Cerney, \& Klosko, 1989) and group-administered (Telch et al., 1993) treatment. In addition, patients completing CBT show marked improvements in overall quality of life (Telch, Schmidt, Jaimez, Jacquin, \& Harrington, 1995). For example, CBT for panic disorder produces marked improvements in familial, social, and vocational quality-of-life indices (Telch et al., 1995).

Little is known, however, about the effects of treatment on physical health outcomes in patients with panic disorder. There is some suggestion that pharmacological treatment for panic disorder may positively affect certain cardiac factors, including heart rate variability (Klein et al., 1995; Tucker et al., 
1997), as well as cardiac conditions such as mitral valve prolapse (Coplan, Papp, King, \& Gorman, 1992). On the other hand, some pharmacotherapy interventions appear to adversely affect cardiovascular functioning (Roth et al., 1992; Taylor \& Hayward, 1990). For example, tricyclic antidepressants appear to increase risk for hypertension (Louie, Louie, \& Lannon, 1992). We could not find studies evaluating the effects of CBT for panic disorder on nonpsychiatric medical outcomes.

In the present study, we sought to extend previous work on the interplay between panic disorder and physical health by examining the effects of CBT for panic disorder on nonpsychiatric medical symptoms. Because CBT has been found to substantially decrease anxiety symptoms and to improve quality of life, and because high levels of anxiety symptoms appear to be related to a variety of nonpsychiatric medical problems, it was hypothesized that treatment-related alleviation of distress would have a significant impact on physical health symptoms. Specifically, patients completing CBT for panic disorder were expected to show fewer nonpsychiatric physical symptoms relative to a wait-list control condition. If CBT did produce positive physical health outcomes, we were also interested in evaluating the role of possible mediating (e.g., decreased anxiety symptoms) and moderating factors (e.g., presence of a chronic nonpsychiatric medical condition) affecting this relationship.

\section{Method}

\section{Participants}

The clinical sample consisted of 46 patients $^{1}$ meeting the following criteria: (a) principal DSM-IV (American Psychiatric Association, 1994) Axis I diagnosis of panic disorder with or without agoraphobia, (b) no change in medication type or dose during the 8 weeks prior to treatment, (c) no evidence of serious suicide intent, (d) no evidence of current substance abuse, and (e) no evidence of current or past schizophrenia, bipolar disorder, or organic mental disorder. Sixty-three percent of the patients were female, with a mean age of 37.9 years $(S D=12.5)$. The majority of these patients were Caucasian $(80.4 \%)$, married $(56.5 \%)$, and employed $(71.7 \%)$. The majority $(71 \%)$ received a diagnosis of panic disorder with agoraphobia and $41 \%$ had at least one co-occurring Axis I diagnosis (e.g., $18 \%$ major depression, $13 \%$ social anxiety disorder). Approximately half of the sample participants were medicated during the study (antidepressants $13 \%$, anxiolytics $28 \%$, both anti-

\footnotetext{
${ }^{1}$ Some of the patients in the present report took part in a study reported previously (Schmidt et al., 2000). Administration of the PHRF was initiated toward the end of recruitment for the Schmidt et al. study such that two groups of patients $(n=12)$ randomized to the CBT group and two groups of patients $(n=10)$ randomized to the delayed treatment group. The present study combines these patients with an additional four groups of patients who were also randomized to CBT (two groups: $n=10$ ) or delayed treatment (two groups: $n=14$ ). PHRF data were not reported in the earlier study.
} 
depressants and anxiolytics 17\%). Physical health status and medical morbidity were not selection criteria.

\section{Procedure}

Patients were selected from applicants who presented for evaluation at an academic research center specializing in the assessment and treatment of anxiety disorders. Diagnostic assessment was based on an initial phone screening interview followed by a face-to-face structured clinical interview using the SCID-NP (First, Spitzer, Gibbon, \& Williams, 1994). Randomly selected videotaped interviews from this sample indicated perfect interrater agreement for a primary diagnosis of panic disorder $(10 / 10)$.

After 5 to 7 treatment-eligible patients had completed the baseline assessment, this "group" was assigned to one of two conditions: CBT or a delayed treatment control. Four groups $(n=22)$ were assigned to the CBT condition and received a group-administered cognitive behavioral treatment that consisted of 12 sessions over a 12-week period (cf. Schmidt et al., 2000). Each session lasted approximately 120 minutes. The treatment protocol included four major components: (a) education and corrective information regarding the etiology and maintenance of panic disorder, (b) cognitive therapy techniques aimed at helping the patient to identify and alter faulty appraisals of threat that contribute to panic occurrence, (c) interoceptive exposure exercises designed to reduce patients' fears of somatic cues through the repeated exposure to feared bodily sensations, and (d) instruction for conducting in vivo exposure exercises designed to reduce patients' fears of external situations through repeated exposure to a fear hierarchy. Patients assigned to the treatment conditions were reassessed immediately following treatment and 6 months after treatment.

Four groups ( $n=24$ ) were assigned to the delayed treatment control condition. Patients in this condition were reassessed after 12 weeks and then received treatment but were not assessed further.

In the active treatment group, treatment integrity was maintained by utilizing a structured and manualized treatment protocol (Safety Maneuver Elimination for Panic Disorder; Schmidt, 1994) that describes the specific goals and strategies for each session. An independent rater's assessment of adherence (Young, Beck, \& Budenz, 1983) to the treatment protocol has yielded $100 \%$ adherence in a randomly selected set of videotaped treatment sessions. The first author administered the treatment to all groups. He is a licensed clinical psychologist with approximately 10 years of experience with cognitive-behavioral treatment of anxiety disorders. In each group, a graduate fellow in clinical psychology, a psychiatry resident, or a psychiatrist acted as co-facilitator.

All 46 subjects who completed the baseline were assessed at posttreatment. All of the patients in the active treatment group completed the treatment (completing at least 11 of 12 sessions). Patients were allowed to reschedule a maximum of 3 sessions with those sessions being rescheduled 
within a few days of the scheduled session. Five of the treated subjects did not complete the 6-month follow-up (they could not be contacted because of address changes). Comparisons between those completing treatment and those lost to follow-up indicated no significant differences in demographics, physical health parameters, and symptom severity at pretreatment or posttreatment $(p s>.05)$.

\section{Measures}

A multimodal assessment battery tapping the major clinical dimensions of panic disorder was administered to all participants at baseline and posttreatment. The same battery was administered to those in the active treatment group at follow-up. Physical health assessments were made at all assessment periods as well as midway during the treatment or waiting period (Week 6 ). One of the anxiety measures was also administered during the midtreatment assessment. Clinician ratings were made by raters masked to treatment condition.

Panic Disorder Severity Scale (PDSS). The PDSS is a semistructured interview rating scale for panic disorder (Shear et al., 1997) that includes ratings of panic frequency and intensity, anticipatory anxiety, avoidance of sensations and situations, and impairment in work and social functioning. Each of these symptoms is rated on a 0 (none) to 4 (extreme) scale. In the present report, a composite score was utilized (i.e., average score of all items). The PDSS has good psychometric properties (Shear et al.). In this sample, two clinicians making PDSS ratings in a dual interview were found to have high reliability (average $r=.91$ ).

Sheehan Patient-Rated Anxiety Scale (SPRAS). The SPRAS (Sheehan, 1983) is a widely used self-report scale for assessing the intensity of anxiety symptoms. The SPRAS has demonstrated adequate test-retest reliability $(r=.67)$ and is highly associated with other measures of anxiety and overall impairment in panic disorder samples (Schmidt, Staab, Trakowski, \& Sammons, 1997). In the present sample the SPRAS shows high internal consistency $(\alpha=.90)$.

General Health Survey (GHS). The GHS measures perceptions about health and includes a checklist for assessing the presence of chronic physical illnesses (e.g., hypertension, asthma, arthritis) and a 6-month history of visits to health professionals. The GHS has been validated with nonpsychiatric and psychiatric samples (McHorney, Ware, Lu, \& Sherbourne, 1994; McHorney, Ware, \& Raczek, 1993). In the present report, we focus only on reported physical illnesses.

Physical Health Rating Form (PHRF). The PHRF is an 11-item, clinicianadministered measure that focuses on the occurrence of physical illness during the past 30 days. Because self-report methods focusing on specific, welloperationalized symptoms show better agreement with physician diagnoses (Orts et al., 1995), patients are given explicit instructions to only endorse specific physical health symptoms arising from medical conditions and to 
exclude symptoms resulting from their anxiety condition. The PHRF covers a number of related domains, including: (a) disability related to physical illness, (b) frequency of physical health symptoms, and (c) perceived physical health status. Questions pertaining to the physical health disability score include: (a) number of days physically ill, (b) number of days missed work or unable to complete normal daily activities as a result of physical illness, (c) number of doctor visits, and (d) number of hospitalizations. Items used to assess frequency of specific symptoms include the number of days experiencing: (a) infections (e.g., flu, common cold), (b) respiratory illness (e.g., asthma), (c) gastrointestinal illness (e.g., ulcer), (d) cardiovascular illness (e.g., MVP), (e) headaches (including migraines), and (f) other illness symptoms. The items targeting specific symptoms were rated on a scale ranging from 0 days to $5+$ days. Finally, the overall perceived physical health rating was made using the following range: excellent $(0)$, very good (1), good (2), fair (3), and poor (4). To score the scale, responses to each item were transformed to range from 0 to 5 such that each item was weighted equally, then summed across the 11 items ( $\alpha=.59$ ).

\section{Results}

\section{Description of Nonpsychiatric Medical Comorbidity and Physical Health Status}

Consistent with previous reports, patients with panic disorder reported a substantial amount of medical comorbidity. Approximately half $(56 \%)$ of all patients reported the presence of at least one chronic physical health condition on the GHS at intake. The most frequently endorsed physical conditions included mitral valve prolapse (17\%), asthma (11\%), thyroid disease (9\%), high blood pressure (9\%), and other (16\%; e.g., reflux, Hepatitis C, various cardiac conditions).

\section{Demographic Comparisons Between Groups}

Baseline differences between CBT-treated patients and delayed-treatment controls were examined using an independent $t$ test for age and chi-square tests for categorical variables (i.e., sex, ethnicity, marital status, and employment status). Participants in the two groups did not significantly differ on any of the demographic variables at baseline $(p s>.05)$.

\section{Pretreatment to Posttreatment Effects of $C B T$ on Anxiety and Physical Health}

In order to establish that the treatment significantly decreased both anxiety symptoms and physical health problems, mixed design analyses of variance (ANOVAs) were conducted with each of three dependent variables. SPRAS and PDSS scores represented anxiety symptoms and PHRF scores represented physical health outcomes. Two time points-pretreatment and post- 
TABLE 1

Group Effects on ANXIety and Health From

Pretreatment to Posttreatment (ANOVAs)

\begin{tabular}{|c|c|c|c|c|c|c|c|}
\hline \multirow[b]{2}{*}{ Group } & \multicolumn{2}{|c|}{ Pre-Treatment } & \multicolumn{2}{|c|}{$\begin{array}{c}\text { Post- } \\
\text { Treatment }\end{array}$} & \multicolumn{3}{|c|}{$F$ Ratios (Partial $\eta^{2}$ ) } \\
\hline & $M$ & $S D$ & $M$ & $S D$ & Group & Time & Interaction \\
\hline \multicolumn{5}{|c|}{ Dependent Variable $=$ SPRAS } & $\begin{array}{c}10.23^{* *} \\
(.19)\end{array}$ & $\begin{array}{c}68.50^{* * * *} \\
(.61)\end{array}$ & $\begin{array}{l}9.67 * * * \\
(.18)\end{array}$ \\
\hline $\mathrm{CBT}$ & 51.09 & 27.77 & 14.36 & 11.67 & & & \\
\hline WL & 60.29 & 29.29 & 43.63 & 18.93 & & & \\
\hline \multicolumn{5}{|c|}{ Dependent Variable $=$ PDSS } & $\begin{array}{c}21.31 * * * \\
(.33)\end{array}$ & $\begin{array}{c}40.36 * * * \\
(.48)\end{array}$ & $\begin{array}{c}15.71 * * * * \\
(.27)\end{array}$ \\
\hline CBT & 1.87 & 0.70 & 0.54 & 0.45 & & & \\
\hline WL & 2.09 & 0.81 & 1.78 & 0.72 & & & \\
\hline \multicolumn{5}{|c|}{ Dependent Variable $=$ PHRF } & $\begin{array}{l}7.31^{*} \\
(.14)\end{array}$ & $\begin{array}{c}10.08^{* * *} \\
(.19)\end{array}$ & $\begin{array}{l}6.64^{* * *} \\
(.13)\end{array}$ \\
\hline $\mathrm{CBT}$ & 10.07 & 5.24 & 4.12 & 3.72 & & & \\
\hline WL & 11.03 & 6.05 & 10.41 & 7.18 & & & \\
\hline
\end{tabular}

Note. Sample size was 22 in the CBT group and 24 in the WL group with the exception of one participant in the WL group missing data on the PDSS. SPRAS = Sheehan PatientRated Anxiety Scale; CBT = cognitive behavioral therapy; WL = wait list; PDSS = Panic Disorder Severity Scale; PHRF = Physical Health Rating Form.

$* p<.05 ; * * p<.01 ; * * * p<.001$.

treatment-composed a within-subjects factor, while group status-CBT or wait list (WL) - was the between-subjects factor in each ANOVA. Table 1 shows descriptive statistics for each outcome variable by time and group as well as the significance and effect sizes of group effects, changes across time, and the interaction of group by time on each outcome.

As noted in Table 1, significant interaction effects emerged for each outcome variable. In addition, all main effects for group and time were significant. Examination of the marginal means reveals that, for each outcome variable, participants in the CBT group began with lower mean scores than participants in the WL group. Regardless of group, mean scores on both of the anxiety measures and the physical health measure decreased across time. However, participants in the CBT group demonstrated significantly (at least at the $p<.01$ level) greater reduction in both anxiety (SPRAS and PDSS) symptoms and physical health problems (PHRF) than participants in the WL group, showing the expected benefit of the treatment on both anxiety and physical health problems. In terms of clinical significance, partial $\eta^{2}$ values for the interactions suggested that being in the CBT group accounted for $13 \%$ of the change in PHRF scores over time, while accounting for $18 \%$ and $27 \%$ of the change in SPRAS and PDSS scores, respectively. 


\section{Mediation and Moderation Analyses}

Mediation. To determine whether the reduction in physical health problems attributed to the group treatment may have been mediated by the reduction in anxiety symptoms, we calculated residualized change scores on the SPRAS from pretreatment to midtreatment, and residualized change scores on the PHRF from midtreatment to posttreatment. The PDSS was not administered at midtreatment and was therefore excluded from analyses of potential mediation. The following four conditions for mediation (Baron \& Kenny, 1986) would be met if the treatment intervention caused a reduction in anxiety and that reduction in anxiety caused a subsequent reduction in physical health problems. First, the treatment would significantly predict the initial change in anxiety. Second, the treatment would also predict the more distal changes in physical health problems. Third, the initial change in anxiety would significantly predict the subsequent change in physical health problems when controlling for the effect of treatment, which, finally, would be substantially reduced compared to its original effect when used alone to predict change in physical health problems.

We conducted two linear regression analyses to evaluate the three conditions for mediation. The results in Table 2 fail to provide evidence for mediation. Although the treatment predicted both SPRAS score reductions from pre- to midtreatment $(\beta=-.48, p<.01)$ and PHRF score reductions from midto posttreatment $(\beta=-.42, p<.05)$, the bottom portion of the table shows that changes in anxiety (residualized SPRAS scores) failed to predict subsequent changes in PHRF scores at the $\alpha=.05$ level $(\beta=.15, p=.41)$ after accounting for the impact of the treatment intervention.

TABLE 2

Linear Regression Results From AnXiety Mediation Analyses

\begin{tabular}{lcclcrrc}
\hline & \multicolumn{2}{c}{ Adjusted } & Predictors & & & \\
Outcome Variable & Step & $R^{2}$ & & Included & \multicolumn{1}{c}{ SE B } & $\beta$ \\
\hline Residual SPRAS & 1 & .20 & Group & -16.08 & 5.16 & $-.48^{* *}$ \\
Residual PHRF & 1 & .15 & Group & -3.79 & 1.45 & $-.42^{*}$ \\
& 2 & .14 & Group & -3.12 & 1.65 & $-.34^{\mathrm{a}}$ \\
& & & Residual SPRAS & .04 & .05 & .15 \\
\hline
\end{tabular}

Note. "Residual SPRAS" refers to the residual values after regressing SPRAS scores at midtreatment on SPRAS scores at pretreatment. "Residual PHRF" refers to the residual values after regressing PHRF scores at posttreatment on PHRF scores at midtreatment. For the treatment group $n=20$ and for the wait-list group $n=15$ due to missing data at the midtreatment collection point. "Group" was coded as follows: cognitive behavioral treatment $=1$, wait list $=0$. SPRAS $=$ Sheehan Patient-Rated Anxiety Scale; PHRF $=$ Physical Health Rating Form.

aApproaches significance, $p<.10$.

${ }^{*} p<.05 ; * * p<.01$. 
Moderation. We conducted a 2 (within subjects, pretreatment versus posttreatment) by 2 (between subjects, CBT or WL group) by 2 (between subjects, presence versus absence of any chronic illness reported in the GHS) mixed design ANOVA to investigate whether the observed impact of the treatment intervention on the reduction of physical health problems depended upon the presence of a chronic illness ( $N=46$ for the analysis, with $n=26$ participants reporting a chronic illness). In the full-factorial model, the threeway interaction of time by group by chronic illness status was not significant at the $\alpha=.05$ level, $F(1,42)=.09, p=.76$. Therefore, no evidence was observed for the presence of a chronic illness affecting the impact of the treatment across time on physical health changes.

\section{Results Through Follow-up for the CBT Group}

Within the CBT group, for which 6-month follow-up data were collected, changes in physical health (PHRF) as well as changes in anxiety (SPRAS) were evaluated across all four time points (pretreatment, midtreatment, posttreatment, and 6-month follow-up) using two univariate repeated measures ANOVAs. Unequal intervals of 5 weeks from pretreatment to midtreatment, 10 weeks from pretreatment to posttreatment, and 36 weeks from pretreatment to 6-month follow-up were specified in the model. Linear, quadratic, and cubic polynomial effects were included. Of the 19 participants who contributed at least some follow-up data, 2 were excluded from the ANOVA examining PHRF scores over time because of missing data on this measure at follow-up, and 2 others were excluded from the ANOVA examining SPRAS scores because of missing data at midtreatment, resulting in $n=17$ for each analysis.

For the polynomial ANOVA examining SPRAS scores over time, overall $F(1.86,29.83)=22.97, p<.001$, after applying a Huynh-Feldt Epislon correction for violation of the sphericity of variance assumption. This indicated an overall effect of time on SPRAS scores. Follow-up tests of polynomial effects showed significant linear, $F(1,16)=29.74, p<.001$, quadratic, $F(1,16)=33.26, p<.001$, and cubic, $F(1,16)=6.02, p<.05$, effects of time on SPRAS scores. In the PHRF ANOVA, time effects were also significant overall, $F(3,48)=23.42, p<.001$, as were follow-up tests of linear, $F(1,16)=30.64, p<.001$, and quadratic, $F(1,16)=30.41, p<.001$, trends. The cubic effect was not significant, $F(1,16)=3.78, p=.07$. Figure 1 depicts the pattern of findings for both outcome measures.

As a further test of the nature of the significant trends, post hoc $t$ tests of all pairwise comparisons across time were conducted within each repeated measures ANOVA, applying a Bonferroni adjustment to the resulting significance levels. For the SPRAS, pretreatment scores differed from scores at each other time point with at least $p<.01$ significance. Pretreatment PHRF scores also differed from scores at each other time point with at least $p<.001$ significance. The remaining time points did not differ from one another (at the $\alpha=$ .05 level) for either outcome measure. Figure 1 shows the decrease in both 


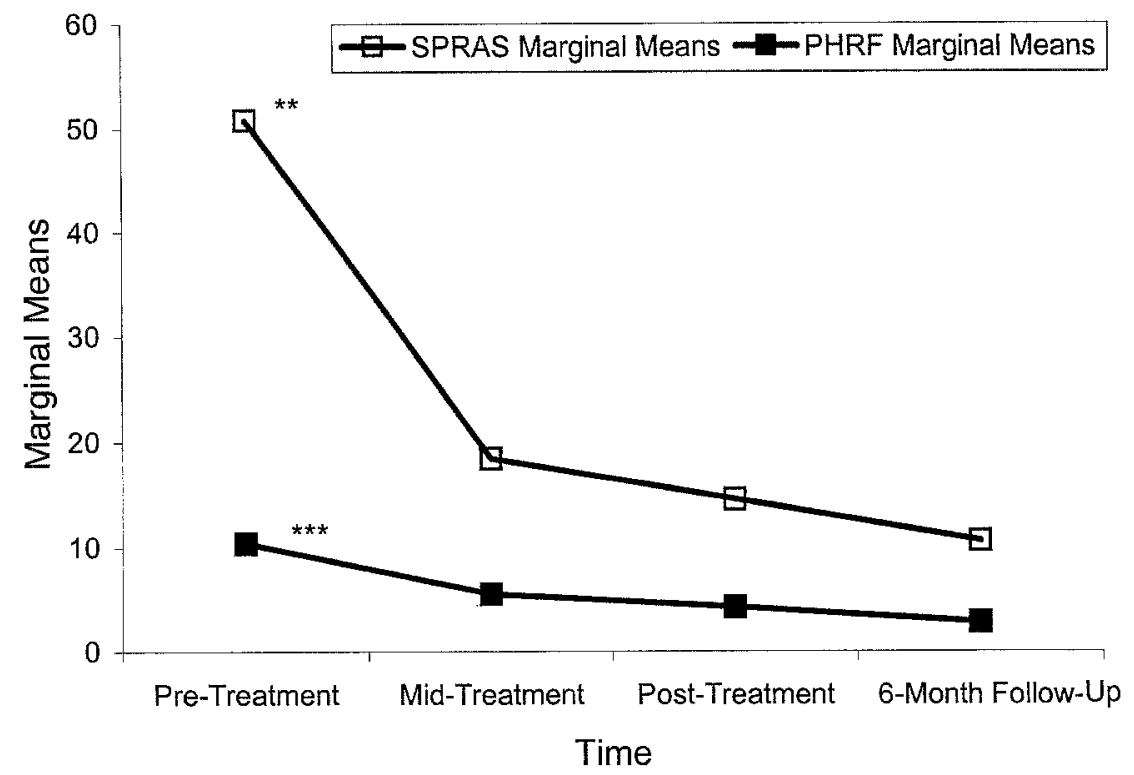

FIG. 1. Sheehan Patient-Rated Anxiety Scale (SPRAS) scores and Physical Health Rating Form (PHRF) scores from pretreatment through follow-up in the cognitive behavioral treatment group ( $n=17$ for each variable). The double and triple asterisks denote that pretreatment scores on the SPRAS and PHRF, respectively, differed from scores at the other three time points with at least $p<.01$ (SPRAS) and $p<.001$ (PHRF) significance, respectively, after Bonferroni correction for all possible comparisons among time points for each variable using $t$ tests. Scores from midtreatment through follow-up did not differ significantly $(p>.05)$ from each other for either measure after Bonferroni adjustment.

anxiety and physical health problems from pretreatment to midtreatment that was maintained throughout the follow-up period.

\section{Discussion}

As far as we are aware, this is the first study to evaluate the effects of cognitive behavioral therapy for panic disorder on physical health status. Consistent with many other studies, we have demonstrated that cognitive behavioral therapy has a significant impact on anxiety symptoms. Of particular interest here, we also found that cognitive behavioral therapy for panic disorder led to improvements on physical health status as measured by a composite selfreport rating scale. The fact that significant changes in physical health status were already evident by midtreatment suggests that CBT can also produce fairly rapid benefits in relation to physical health.

Interestingly, mediation analyses indicated that changes in anxiety symptoms did not account for the relationship between treatment and the physical health variables at posttreatment. This would suggest that treatment may be 
affecting physical health through some other mechanism. One possibility is that general affective distress or depression may be acting as a mediating variable. This alternative hypothesis is consistent with the fact that CBT has a notable impact on depression symptoms and that depression has a negative impact on physical health (Badger, 1998; Druss, Allen, \& Bruce, 1998; Judd, Paulus, Wells, \& Rapaport, 1996). To investigate this, we conducted additional post hoc mediation analyses evaluating changes in depression symptoms as a potential mediator variable. However, these analyses did not yield convincing evidence of mediation. Taken together, there is little evidence to suggest that changes in distress mediated the relationship between CBT and physical health.

An alternative hypothesis is that the changes noted in physical health status may be a result of changes in psychological or cognitive factors that influence perceptions of physical health symptoms. Attention or vigilance to bodily sensations has taken on increased importance in psychological conceptualizations of panic. For example, cognitive models of panic (Barlow, 1988; Clark, 1986) suggest that patients may become vigilant to bodily arousal as a consequence of experiencing panic attacks. Related to this, patients with panic disorder appear to have greater interoceptive accuracy (Ehlers \& Breuer, 1992). In fact, some of our earlier work confirms the fact that patients with panic disorder are more consciously vigilant to bodily sensations relative to controls and other anxiety disorders (Schmidt, Lerew, \& Trakowski, 1997). Conceivably, increased interoceptive acuity or increased attention to bodily cues may result in increased reporting of physical health symptoms. CBT protocols for panic disorder attempt to target interoceptive processes (e.g., Barlow \& Craske, 2000). Moreover, Schmidt, Lerew, et al. (1997) found that vigilance to bodily sensations was significantly diminished following CBT for panic disorder. Therefore, patients in the present study may simply be less aware of physical symptoms that they had previously attributed to a medical condition. Unfortunately, it is impossible to know whether the treatment actually influenced physical health status or whether the treatment simply altered perceptions of physical health in the present study. Future work involving biological parameters is critical to more fully address this issue.

An important consideration regarding the study findings is that nonpsychiatric medical morbidity was based on patient self-report instead of examinations by physicians or laboratory methods. Self-report of physical symptoms as opposed to health measures obtained from a comprehensive physical examination is an obvious methodological limitation that leaves open the question of whether patients with panic disorder can reliably report nonpsychiatric medical symptoms. Despite potential problems, self-reported health symptoms have been shown to be highly associated with actual health status (LaRue, Bank, Jarvik, \& Hetland, 1979; Mossey \& Shapiro, 1982). For example, Bradford, Graham, and Reinert (1993) found that only $4 \%$ of their patient sample had significant discrepancies between self-reported medical conditions and documented medical histories. Thus, there is reason to believe that the self-report methodology used in the present study is likely to adequately represent 
physical health symptoms. However, it is recommended that additional work exploring this relationship should utilize objective physical health measures.

It is also important to consider that subjective or perceived physical health often provides an accurate gauge of physical health outcomes. A number of prospective reports have found that perceived health predicts mortality (Idler \& Angel, 1990; Idler \& Kasl, 1991; Idler, Kasl, \& Lemke, 1990; Kaplan \& Camacho, 1983; Mossey \& Shapiro, 1982). In fact, perceived physical health has been found to predict mortality better than the presence of health problems (Idler \& Kasl) or health status assessed by physicians (Mossey \& Shapiro). There is also evidence to suggest that perceived health and actual disease severity are independently related to illness-specific anxiety symptoms. For example, Carr, Lehrer, and Hochron (1995) found that asthma patients' subjective reports of asthma symptomatology and pulmonary function tests independently predicted asthma-related panic fear. Thus, perceived physical health may predict consequential mental and physical health outcomes in patients with panic disorder.

The limitations of this study need to be considered. The sample size was modest, particularly for the follow-up analyses. Because some of the findings amount to accepting the null hypothesis (e.g., lack of moderation effects), it is possible that the effects are a result of low statistical power. Therefore, replication is certainly warranted. Another limitation of the present report involved collapsing across many different forms of health problems. Because of the sample size in the present study, it is not feasible to separately evaluate each specific medical condition. This type of measurement approach may obscure potentially salient effects. For example, it may be that CBT differentially affects milder versus more severe medical conditions. It is also important to note that the majority of identified medical problems in the present study were relatively mild (e.g., MVP, high blood pressure). Other samples containing a preponderance of more severe medical conditions (e.g., COPD, heart disease) may produce a different pattern of findings.

The present study points to the complex interplay between panic disorder and physical health outcomes. Consistent with expectations, data indicate that CBT affects mental health and physical symptoms. Future work utilizing biological indicators is needed, however, to more definitively demonstrate this effect.

\section{References}

American Psychiatric Association. (1994). Diagnostic and statistical manual of mental disorders (4th ed.). Washington, DC: Author.

Andreoli, A., Keller, S. E., Rabaeus, M., Zaugg, L., Garrone, G., \& Taban, C. (1992). Immunity, major depression and panic disorder comorbidity. Biological Psychiatry, 31, 896-908.

Badger, T. A. (1998). Depression, physical health impairment and service use among older adults. Public Health Nursing, 15, 136-145.

Barlow, D. H. (1988). Anxiety and its disorders: The nature and treatment of anxiety and panic. New York: The Guilford Press. 
Barlow, D. H., \& Craske, M. G. (2000). Mastery of your anxiety and panic (3rd ed.). San Antonio, TX: Graywind/Psychological Corporation.

Barlow, D. H., Craske, M. G., Cerney, J. A., \& Klosko, J. S. (1989). Behavioral treatment of panic disorder. Behavior Therapy, 20, 261-282.

Baron, R. M., \& Kenny, D. A. (1986). The moderator-mediator variable distinction in social psychological research: Conceptual, strategic, and statistical considerations. Journal of Personality and Social Psychology, 51, 1173-1182.

Bradford, V. P., Graham, B. P., \& Reinert, K. G. (1993). Accuracy of self-reported health histories: A study. Military Medicine, 158, 263-265.

Cacioppo, J. T. (1993). Social neuroscience: Autonomic, neuroendocrine, and immune response to stress. Psychophysiology, 31, 113-128.

Carr, R. E., Lehrer, P. M., \& Hochron, S. M. (1995). Predictors of panic-fear in asthma. Health Psychology, 14, 421-426.

Clark, D. M. (1986). A cognitive approach to panic. Behaviour Research and Therapy, 24, 461-470.

Coplan, J. D., Papp, L. A., King, D. L., \& Gorman, J. M. (1992). Amelioration of mitral valve prolapse after treatment for panic disorder. American Journal of Psychiatry, 149, 1587-1588.

Coplan, J. D., Tamir, H., Calaprice, D., DeJesus, M., de la Nuez, M., Pine, D., Papp, L. A., Klein, D. F., \& Gorman, J. M. (1999). Plasma anti-serotonin and serotonin anti-idiotypic antibodies are elevated in panic disorder. Neuropsychopharmacology, 20,386-391.

Druss, B. G., Allen, H. M., \& Bruce, M. L. (1998). Physical health, depressive symptoms, and managed care enrollment. American Journal of Psychiatry, 155, 878-882.

Ehlers, A., \& Breuer, P. (1992). Increased cardiac awareness in panic disorder. Journal of Abnormal Psychology, 101, 371-382.

First, M. B., Spitzer, R. L., Gibbon, M., \& Williams, J. B. (1994). Structured clinical interview for DSM-IV-Patient Edition (SCID-N/P, Version 2.0). New York: Biometrics Research Department, New York State Psychiatric Institute.

Glaser, R., Kennedy, S., Lafuse, W. P., Bonneau, R. H., Speicher, C. E., Hillhouse, J., \& Kiecolt-Glaser, J. K. (1990). Psychological stress-induced modulation of interleukin 2 receptor gene expression and interleukin 2 production in peripheral blood leukocytes. Archives of General Psychiatry, 47, 707-712.

Gorman, J. M., Goetz, R. R., Fyer, M., King, D. L., Fyer, A. J., Liebowitz, M. R., \& Klein, D. F. (1988). The mitral valve prolapse-panic disorder connection. Psychosomatic Medicine, 50, 114-122.

Idler, E. L., \& Angel, R. J. (1990). Self-rated health and mortality in the NHANES-I epidemiologic follow-up study. American Journal of Public Health, 80, 446-452.

Idler, E. L., \& Kasl, S. (1991). Health perceptions and survival: Do global evaluations of health status really predict mortality? Journal of Gerontology, 46, S55-S65.

Idler, E. L., Kasl, S., \& Lemke, J. H. (1990). Self-evaluated health and mortality among the elderly in New Haven, Connecticut, and Iowa and Washington Counties, Iowa, 1982-1986. American Journal of Epidemiology, 131, 91-103.

Judd, L. L., Paulus, M. P., Wells, K. B., \& Rapaport, M. H. (1996). Socioeconomic burden of subsyndromal depressive symptoms and major depression in a sample of the general population. American Journal of Psychiatry, 153, 1411-1417.

Kahn, J. P., Drusin, R. E., \& Klein, D. F. (1987). Idiopathic cardiomyopathy and panic disorder: Clinical association in cardiac transplant candidates. American Journal of Psychiatry, 144 , $1327-1330$.

Kaplan, G. A., \& Camacho, T. (1983). Perceived health and mortality: Nine-year follow-up of the Human Population Laboratory cohort. American Journal of Epidemiology, 117, 292-304.

Karajgi, B., Rifkin, A., Doddi, S., \& Kolli, R. (1990). The prevalence of anxiety disorders in patients with chronic obstructive pulmonary disease. American Journal of Psychiatry, 147, 200-201.

Kawachi, I., Colditz, G. A., Ascherio, A., Rimm, E. B., Giovannucci, E., Stampfer, M. J., \& 
Willett, W. C. (1994). Prospective study of phobic anxiety and risk of coronary heart disease in men. Circulation, 89, 1992-1997.

Kiecolt-Glaser, J. K., \& Glaser, R. (1995). Psychoneuroimmunology and health consequences: Data and shared mechanisms. Psychosomatic Medicine, 57, 269-274.

Klein, E., Cnaani, E., Harel, T., Braun, S., \& Ben-Haim, S. A. (1995). Altered heart rate variability in panic disorder patients. Biological Psychiatry, 37, 18-24.

LaRue, A., Bank, L., Jarvik, L., \& Hetland, M. (1979). Health in old age: How do physicians' ratings and self-ratings compare? Journal of Gerontology, 34, 687-691.

Louie, A. K., Louie, E. K., \& Lannon, R. A. (1992). Systemic hypertension associated with tricyclic antidepressant treatment in patients with panic disorder. American Journal of Cardiology, 70, 1306-1309.

Marazziti, D., Ambrogi, F., Vanacore, R., Mignani, V., Savino, M., Palego, L., Cassano, G. B., \& Akiskal, H. S. (1992). Immune cell imbalance in major depressive and panic disorders. Neuropsychobiology, 26, 23-26.

McCue, E. C., \& McCue, P. A. (1984). Organic and hyperventilatory causes of anxiety-type symptoms. Behavioural Psychotherapy, 12, 308-317.

McHorney, C. A., Ware, J. E., Jr., Lu, J. F. R., \& Sherbourne, C. D. (1994). The MOS 36-Item Short-Form Health Survey (SF-36): III. Tests of data quality, scaling assumptions, and reliability across diverse patient groups. Medical Care, 32, 40-66.

McHorney, C. A., Ware, J. E., Jr., \& Raczek, A. E. (1993). The MOS 36-Item Short-Form Health Survey (SF-36): II. Psychometric and clinical tests of validity in measuring physical and mental health constructs. Medical Care, 31, 247-263.

Mossey, J. M., \& Shapiro, E. (1982). Self-rated health: A predictor of mortality among the elderly. American Journal of Public Health, 71, 800-808.

Orts, K., Sheridan, J. F., Robinson-Whelen, S., Glaser, R., Malarkey, W. B., \& Kiecolt-Glaser, J. K. (1995). The reliability and validity of a structured interview for the assessment of infectious illness symptoms. Journal of Behavioral Medicine, 18, 517-529.

Raj, B. A., Corvea, M. H., \& Dagon, E. M. (1993). The clinical characteristics of panic disorder in the elderly: A retrospective study. Journal of Clinical Psychiatry, 54, 150-155.

Rapaport, M. H. (1998). Circulating lymphocyte phenotypic surface markers in anxiety disorder patients and normal volunteers. Biological Psychiatry, 43, 458-463.

Rapaport, M. H., \& Stein, M. B. (1994). A preliminary study of serum cytokine and soluble interleukin-2 receptors in patients with panic disorder. Anxiety, 1, 22-25.

Rogers, M. P., White, K., Warshaw, M. G., Yonkers, K. A., Rodriguez-Villa, F., Chang, G., \& Keller, M. B. (1994). Prevalence of medical illness in patients with anxiety disorders. International Journal of Psychiatry in Medicine, 24, 83-96.

Roth, W. T., Margraf, J., Ehlers, A., Haddad, J. M., Maddock, R. J., Agras, W. S., \& Taylor, C. B. (1992). Imipramine and alprazolam effects of stress test reactivity in panic disorder. Biological Psychiatry, 31, 35-51.

Schmidt, N. B. (1994). Safety maneuver elimination for panic disorder. Unpublished treatment manual.

Schmidt, N. B., Lerew, D., \& Trakowski, J. J. (1997). Body vigilance in panic disorder: Evaluating attention to bodily perturbations. Journal of Consulting and Clinical Psychology, 65, 214-220.

Schmidt, N. B., Staab, J. P., Trakowski, J. H., \& Sammons, M. (1997). Efficacy of a brief psychosocial treatment for panic disorder in an active duty sample: Implications for military readiness. Military Medicine, 162, 123-129.

Schmidt, N. B., Woolaway-Bickel, K., Trakowski, J., Santiago, H., Storey, J., Koselka, M., \& Cook, J. (2000). Dismantling cognitive-behavioral treatment for panic disorder: Questioning the utility of breathing retraining. Journal of Consulting and Clinical Psychology, 68, 417-424.

Shear, M. K., Brown, T. A., Barlow, D. H., Money, R., Sholomskas, D. E., Woods, S. W., Gorman, J. M., \& Papp, L. A. (1997). Multicenter collaborative panic disorder severity scale. American Journal of Psychiatry, 154, 1571-1575. 
Sheehan, D. V. (1983). The anxiety disease. New York: Scribners.

Taylor, C. B., \& Hayward, C. (1990). Cardiovascular considerations in selection of anti-panic pharmacotherapy. Journal of Psychiatric Research, 24, 43-49.

Telch, M. J., Lucas, J. A., Schmidt, N. B., Hanna, H. H., Jaimez, T. L., \& Lucas, R. (1993). Group cognitive-behavioral treatment of panic disorder. Behaviour Research and Therapy, $31,279-287$.

Telch, M. J., Schmidt, N. B., Jaimez, L., Jacquin, K., \& Harrington, P. (1995). The impact of cognitive-behavioral therapy on quality of life in panic disorder patients. Joumal of Consulting and Clinical Psychology, 63, 823-830.

Tucker, P., Adamson, P., Miranda, R., Scarborough, A., Williams, D., Groff, J., \& McLean, H. (1997). Paroxetine increases heart rate variability in panic disorder. Journal of Clinical Psychopharmacology, 17, 370-376.

Weissman, M. M., Markowitz, J. S., Ouellette, R., Greenwald, S., \& Kahn, J. P. (1990). Panic disorder and cardiovascular/cerebrovascular problems: Results from a community survey. American Journal of Psychiatry, 147, 1504-1508.

Wells, K. B., Golding, J. M., \& Burnam, M. A. (1989). Chronic medical conditions in a sample of the general population with anxiety, affective, and substance use disorders. American Journal of Psychiatry, 146, 1440-1446.

Wolfe, B. E., \& Maser, J. E. (Eds.). (1994). Treatment of panic disorder: A consensus development conference. Washington, DC: American Psychiatric Press.

Young, J., Beck, A. T., \& Budenz, D. (1983). Assessment of compliance in cognitive therapy. Unpublished manuscript, University of Pennsylvania.

Zandbergen, J., Bright, M., Pols, H., Fernandez, I., deLoof, C., \& Griez, E. J. (1991). Higher lifetime prevalence of respiratory diseases in panic disorder? American Journal of Psychiatry, 148, 1583-1585.

ReCeIVED: January 23, 2002

ACCEPTED: July 29, 2002 